\title{
Electrochemical Studies and Molecular Dynamics Simulation of the Interaction between Accelerators and Cu Surface During the Electroplating Process
}

\author{
Zhongliang Xiao, Zhaohua Zhou, Liubin Song, DaoXin Wu*, Chao Zeng, Zhong Cao \\ Hunan Provincial Key Laboratory of Materials Protection for Electric Power and Transportation, \\ School of Chemistry and Biological Engineering, Changsha University of Science and Technology, \\ Hunan Changsha 410114, PR China. \\ *E-mail: xiaozhongliang@163.com
}

doi: $10.20964 / 2019.05 .25$

Received: 2 January 2019 / Accepted: 4 March 2019 / Published: 10 April 2019

Accelerators are crucial for electroplating in the electronics industry. In this research, the electrochemical behavior of two accelerators, 3-(benzothiazolyl-2-mercapto)-propyl-sulfonic acid sodium salt (ZPS) and bis(3-sulfopropyl) disulfide (SPS), during $\mathrm{Cu}$ electrodeposition was analyzed using electrochemical techniques. Meanwhile, the adsorption behavior of the two accelerators on $\mathrm{Cu}(111)$ was also studied using molecular dynamics (MD) simulation, which predicts the preferable reaction sites for nucleophilic or electrophilic attack and the corresponding interactions based on density functional theory. The MD results showed that ZPS could be adsorbed on the $\mathrm{Cu}(111)$ firmly through the thiouronium group and a benzothiazolyl group, whereas the SPS was adsorbed through the thiouronium group. Moreover, the accelerating efficiency of these two accelerators was closely related to the frontier molecule orbital density distributions and Fukui indices.

Keywords: copper electroplating; electrochemistry; MD simulation; ZPS; SPS

\section{$\underline{\text { FULL TEXT }}$}

(C) 2019 The Authors. Published by ESG (www.electrochemsci.org). This article is an open access article distributed under the terms and conditions of the Creative Commons Attribution license (http://creativecommons.org/licenses/by/4.0/). 